\title{
Infants show stability of goal-directed imitation
}

\author{
Elena Sakkalou $^{\mathrm{a}, \mathrm{b}}$, Kate Ellis-Davies ${ }^{\mathrm{a}}$, Nia C. Fowler ${ }^{\mathrm{a}}$, Elma E. Hilbrink ${ }^{\mathrm{a}, \mathrm{c}}$, \\ Merideth Gattis ${ }^{\mathrm{a}, *}$

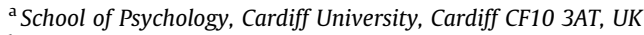 \\ ${ }^{\mathrm{b}}$ Neurosciences Unit, UCL Institute of Child Health, London WC1N 3LU, UK \\ ${ }^{\mathrm{c}}$ Max Planck Institute for Psycholinguistics, 6525 XD Nijmegen, The Netherlands
}

\section{A R T I C L E I N F O}

\section{Article history:}

Received 31 August 2011

Revised 9 September 2012

Available online 13 October 2012

\section{Keywords:}

Imitation

Goal understanding

Individual differences

Action understanding

Prosody

Infancy

Longitudinal

\begin{abstract}
A B S T R A C T
Previous studies have reported that infants selectively reproduce observed actions and have argued that this selectivity reflects understanding of intentions and goals, or goal-directed imitation. We reasoned that if selective imitation of goal-directed actions reflects understanding of intentions, infants should demonstrate stability across perceptually and causally dissimilar imitation tasks. To this end, we employed a longitudinal within-participants design to compare the performance of 37 infants on two imitation tasks, with one administered at 13 months and one administered at 14 months. Infants who selectively imitated goal-directed actions in an object-cued task at 13 months also selectively imitated goal-directed actions in a vocal-cued task at 14 months. We conclude that goal-directed imitation reflects a general ability to interpret behavior in terms of mental states.
\end{abstract}

(c) 2012 Elsevier Inc. All rights reserved.

\section{Introduction}

A variety of studies have demonstrated that infants view others as intentional agents, interpreting their actions as goal-directed (e.g., Baldwin, Baird, Saylor, \& Clark, 2001; Phillips, Wellman, \& Spelke, 2002; Woodward, 1998). Around their first birthday, infants begin to act on this knowledge, selectively imitating the goals and intentions of others (Brugger, Lariviere, Mumme, \& Bushnell, 2007; Carpenter, Akhtar, \& Tomasello, 1998; Carpenter, Call, \& Tomasello, 2005; Meltzoff, 1995; Nielsen, 2009). For example, Carpenter and colleagues (2005) showed 12- and 18-month-olds a game in which an experimenter moved a toy mouse across a mat. In one condition, the mouse hopped to one of two

\footnotetext{
* Corresponding author. Fax: +44 2920874858.

E-mail address: gattism@cardiff.ac.uk (M. Gattis).
} 
houses. In another condition, no houses were present; the experimenter made the same motions and placed the mouse in the same, but house-less, location. At both ages, infants were more likely to place the mouse in the same location as the experimenter when houses were present and more likely to move the mouse with the same motion as the experimenter when no houses were present. Because infants in both conditions observed identical actions but selectively copied different aspects of those actions depending on whether or not a house was present, Carpenter and colleagues concluded that infants interpret people's actions in terms of a goal hierarchy and selectively imitate the most important goals. This pattern of learning, also observed in children and adults, has been called goal-directed imitation (Bekkering, Wohlschläger, \& Gattis, 2000; Gattis, Bekkering, \& Wohlschläger, 2002).

Some researchers have argued, however, that infants' imitative behavior does not involve inferences about mental states, such as goals and intentions, but instead results from learning about specific outcomes. For example, in a seminal study, Meltzoff (1995) reported that infants were just as likely to perform a target action after seeing a failed attempt at that action as after seeing the complete action, and he concluded that infants perceive and imitate the actions of others in terms of goals and intentions. Huang, Heyes, and Charman (2002) argued against this interpretation, proposing that completing an incomplete action did not require intention attribution. They compared infant imitation after a complete target action, a failed attempt, and before-and-after scenes with the same objects but no actions. Infants were just as likely to perform the target action following the before-and-after scenes as following the failed attempt. Because both conditions involved incomplete information but only one involved human actions, Huang and colleagues argued that infants were learning about and imitating outcomes rather than intentions.

Other researchers have argued that, rather than drawing inferences about goals or intentions, infants are responding to specific cues such as object affordances and communicative cues. Carpenter and colleagues (2005) considered that in their study when infants placed the mouse in the house, they might have been responding to object affordances; placing the mouse inside the house may have been a response to the containment properties of the house or, in other words, the cues of the object itself. Other researchers have argued that, rather than relying on inferences about mental states to choose what to imitate, infants rely on specific communicative cues such as eye contact, infant-directed speech, and indicative phrases such as "Look!" (Gergely \& Csibra, 2005). To support this claim, researchers point toward evidence that infants are less likely to imitate a novel action when the adult demonstrator leaves the room (Kiraly, 2009).

In this article, we report a study comparing infant imitation across two different tasks to investigate whether imitation reflects a general ability to interpret others' behavior in terms of mental states or instead reflects learning about specific outcomes or attention to specific cues. Our study drew on the example of Sommerville and Woodward (2005), who demonstrated a link between comprehension and production of goal-directed behavior by comparing 10-month-olds' performance across two tasks. They assessed sensitivity to others' goals by comparing looking times across different contexts in which an adult reached for a toy. As a group, 10-month-olds were not sensitive to others' goals. Importantly, however, performance on the comprehension task was related to performance on a similar production task; infants who looked longer at new goals than at new strategies, or means, also produced more goal-directed strategies themselves. Sommerville and Woodward noted that individual differences on the two tasks were critical to identifying this relation and that these individual differences were observable at an age when the ability of interest was not yet observed at the group level.

Whereas Sommerville and Woodward (2005) were interested in whether a general ability to represent behavior in terms of intentions might govern both perception and action and so chose tasks that were similar but involved different responses from infants, we were interested in whether a general ability to represent behavior in terms of intentions might govern imitation across a variety of contexts and so chose two tasks that were dissimilar but always involved imitative responses. The two tasks differed in terms of physical objects, goals, and the cues that might signal goals. This last difference was particularly important because it allowed us to test whether infant imitation relies on a general ability to infer intentions or on attention to specific cues and outcomes.

An object-cued task, based on the mouse task of Carpenter and colleagues (2005), was used to measure imitation at 13 months. This task manipulated the presence or absence of certain objects across 
conditions, but the actions and communicative cues remained the same. Including this task allowed us to examine the possibility that object properties, such as the containment properties of the house, rather than inferences about mental states might determine infant responses.

A vocal-cued task was used to measure imitation at 14 months. In the vocal-cued task, the experimenter performed a two-action sequence on a novel toy and used prosodic cues to mark one action as intentional and the other action as accidental. In one study using this paradigm, the experimenter marked actions with "There!" or "Whoops!" and the corresponding prosody of intentions or accidents (Carpenter et al., 1998). In a more recent study, the experimenter used novel words marking intentions versus accidents with prosody alone (Sakkalou \& Gattis, 2012). In both studies infants copied intentional actions more than accidental actions, and in both studies the authors concluded that imitation is a selective process based on inferences about the goals of others. Sakkalou and Gattis reported that at the group level, infants did not demonstrate this pattern until 16 months but that some infants did so at 14 months. In contrast to the object-cued task, the vocal-cued task manipulated communicative cues; actions and objects were counterbalanced, so that the critical contrast was prosody signaling mental states. Including this task allowed us to examine the possibility that communicative cues, rather than inferences about mental states, might determine infant responses.

The two tasks were administered to the same infants in a longitudinal design, with the object-cued task at 13 months and the vocal-cued task at 14 months. This design was based on previous findings demonstrating that (a) before the first birthday, infants represent the behavior of others in terms of intentions (e.g., Woodward, 1998); (b) around the first birthday, this ability begins to guide imitation across a variety of contexts, leading infants to selectively imitate the goals and intentions of others (e.g., Carpenter et al., 1998, 2005); and (c) examining individual differences across tasks during a transitional period for an ability allows underlying relations to be identified (e.g., Sommerville \& Woodward, 2005). We reasoned that if goal-directed imitation relies on a general ability to make inferences about mental states, infants who demonstrate goal-directed imitation on one task should be more likely to demonstrate goal-directed imitation on the other task despite the differences in perceptual and causal structure across the tasks. In contrast, if goal-directed imitation is merely a consequence of infant attention to object affordances or communicative cues, performance on each task should be more independent and, therefore, we would not expect to observe stability across the two tasks.

\section{Method}

\section{Participants}

A total of 37 infants ( 19 boys and 18 girls) were tested on the object-cued task at 13 months $(M=395$ days, range $=389-410)$ and on the vocal-cued task at 14 months $(M=427$ days, range $=412-441$ days). No infants were excluded from analyses. The reported study was part of the First Steps project, a longitudinal study of healthy infant development from birth to 18 months (Ellis-Davies, Sakkalou, Fowler, Hilbrink, \& Gattis, in press). At each monthly testing session, families were given $£ 25$ in shopping vouchers and a small toy for their participation.

\section{Material and apparatus}

\section{Object-cued task}

Because Carpenter and colleagues (2005) excluded a large number of infants due to noncompliance and participation from all infants was essential to our study design, we adapted the task to increase compliance. The adapted task included (a) three toy animals; (b) positioning the final location at the end of the board rather than the middle to give more emphasis to the direction of movement; and (c) a third condition, analogous to the house condition, in which the experimenter placed the toy on one of two towers. Object trials involved two objects (houses or cylinders painted to resemble towers) mounted at the end of a wooden board, and No-Object trials involved a wooden board. All trials involved the same three toy animals. 
Vocal-cued task

Five novel toys were constructed from wooden boxes and hardware items. Each toy had two objects mounted on it such as a handle and a pull cord. An interesting end result, such as a puppet dancing, followed after the experimenter performed the second action.

\section{Design and procedure}

\section{Object-cued task}

The within-participants design involved two Object trials and one No-Object trial. On each trial, the experimenter said, "Look what I can do with this one," grasped one of three animals, and made it hop to the other end of the board, making a nonword vocalization while doing so (e.g., "tat, tat, tat"). The hopping action was the same for all trials, with the only difference being whether the final location was the left or right side of the end of the board. In the Object conditions, the animal was placed in one of the houses or on one of the towers. In the No-Object condition, the animal was placed in one of the same, but object-less, locations. Following each demonstration, the three animals were placed at the front of the board and infants were told, "It is your turn now." If an infant did not respond within 45 to $60 \mathrm{~s}$, the experimenter proceeded to the next condition. The chosen animal, vocalization, final location (left or right), and order of condition (Object-House, Object-Tower, or No-Object) were counterbalanced across participants.

\section{Vocal-cued task}

The within-participants design involved one warm-up trial and four experimental trials. The warm-up trial involved just one action and no vocalization. On each experimental trial, the experimenter directed children's attention to the toy, performed one action followed by a vocalization, and then after a short delay performed a second action followed by a vocalization. After the second action, the end result appeared. Infants were then told, "It's your turn; can you make it work?" Infants participated in two trials for each of the four boxes for a total of eight trials. The experimenter maintained a neutral facial expression throughout each trial. During the infant response period, the end result occurred only if infants produced the intentional action but regardless of whether infants also produced the accidental action (i.e., the end result followed when infants performed the intentional action only or performed both actions together). Following Sakkalou and Gattis (2012), accidental vocalizations had a rising contour and intentional vocalizations had a falling contour expressed through the Greek words "Ochi" and "Nato." These foreign language words were novel, and therefore meaningless, to the participants. The pairing of prosodic contour and word was counterbalanced across participants, so that for half of the infants "Ochi" was accidental and "Nato" was intentional and vice versa. Two orders of action sequence alternated across trials: I-A (first intentional and then accidental) and A-I (first accidental and then intentional).

\section{Coding and reliability}

\section{Object-cued task}

For each of the three trials, a trained and hypothesis-blind coder scored from video all instances of hopping (motion matches) and placing the animal in the end location (location matches). Matches of motion meant that infants needed to produce two or more hopping motions. Matches of location meant that infants needed to put the animal in the house and on the tower in the Object conditions and place the animal on the far end of the wooden area (defined as the last one-third) in the No-Object condition. Motion matching was defined as selective matching of hopping movements toward any trajectory even if it was not in the same trajectory as the one demonstrated. Location matching was defined as selective matching of location even if infants did not put the animal in the same side as the one demonstrated. To assess reliability, the experimenter scored eight videos. Interrater reliability was assessed using Cohen's kappa, which showed perfect agreement $(\kappa=1.00)$. 
Vocal-cued task

For each of the eight trials, the experimenter scored from video whether infants reproduced the first action, the second action, both actions in the demonstrated order, both actions in a reversed order, or neither action. The numbers of intentional and accidental actions were calculated from this depending on whether the first or second action was intentional or accidental. Each time infants performed the intentional action, they received a score of 1 in the intentional action category. Each time they performed the accidental action, they received a score of 1 in the accidental action category. To assess reliability, a trained and hypothesis-blind secondary coder scored eight videos. Interrater reliability was assessed using Cohen's kappa, which showed high agreement $(\kappa=.98)$.

\section{Results}

\section{Object-cued task performance}

First, we examined the effect of counterbalancing on location and motion matching. One-way analyses of variance (ANOVAs) showed no effect of counterbalancing order, $F(5,31)=1.46, p=.23$, and $F(5,31)=0.79, p=.56$, respectively. To address possible learning across the task, we compared the frequency of location, $Q(1)=.09, p=.76$, and motion, $Q(1)=.00, p=1.00$, in the first trial versus third trial. The results revealed no difference between trials.

The next analyses investigated goal-directed imitation at the group level. A Wilcoxon pairedsamples test showed that in both Object conditions, infants produced significantly more location matches (House: $M=.35, S D=.48$; Tower: $M=.30, S D=.46$ ) than motion matches (House: $M=.08$, $S D=.28$; Tower: $M=.11, S D=.31), z=2.50, p<.05$, and $z=2.11, p<.05$, respectively. In the No-Object condition, infants produced significantly more motion matches $(M=.19, S D=.40)$ than location matches $(M=.03, S D=.16), z=2.12, p<.05$. No significant difference was observed between the two final Object conditions for location, $z=.50, p=.62$, and motion, $z=.38, p=.71$. These group-level results replicated those of Carpenter and colleagues (2005).

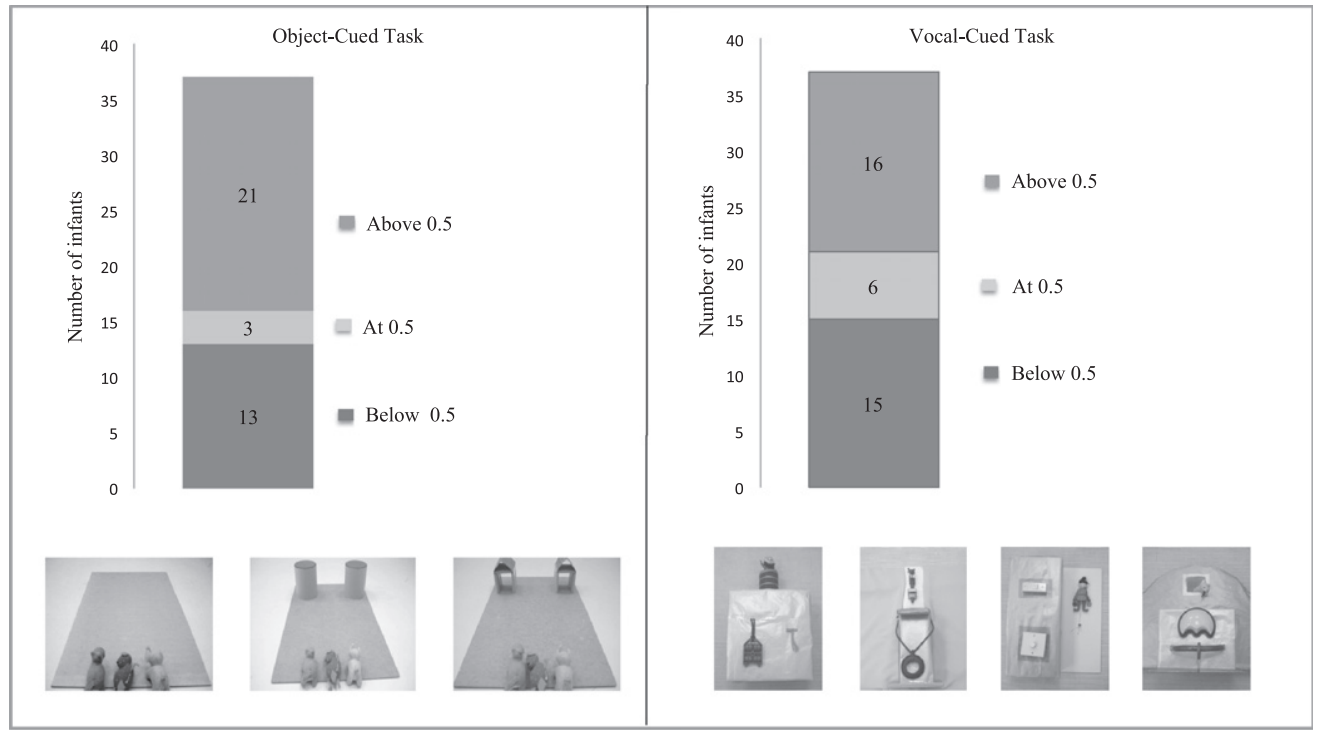

Fig. 1. Proportions of goal-directed imitation in the object-cued task (left) and vocal-cued task (right). The bars display the numbers of infants who performed a greater number of goal imitation (above 0.5), an equal number of goal imitation and other imitation (at 0.5), and a greater number of other imitation (below 0.5). Depicted below the graphs are photographs of the stimuli used in the two tasks. Note: Of the 13 infants who scored a proportion below 0.5 on the object-cued task, 10 produced no behaviors. This included infants who may have taken the animal toys but produced no actions with them. 
Vocal-cued task performance

We first examined the effect of lexical order on the number of intentional actions. The results showed no effect of lexical order, $F(1,35)=0.23, p=.63$. Therefore, the data were collapsed for subsequent analyses.

A Cochran $Q$ test showed no effect of learning throughout the task. Infants produced similar frequencies of intentional actions in the first and eighth trials, $Q(1)=.05, p=.82$.

The next analyses investigated goal-directed imitation at the group level. A Wilcoxon paired-samples test did not indicate that infants produce significantly more intentional actions $(M=5.41$, $S D=1.82)$ than accidental actions $(M=5.05, S D=1.97), z=.71, p=.48$. These group-level results were consistent with those of Sakkalou and Gattis (2012).

\section{Relation between performances on object-cued task and vocal-cued task}

To investigate whether individual performance on the two imitation tasks was related, we conducted a correlational analysis. For both tasks, a proportional score for goal-directed imitation was calculated by comparing goal-directed imitation with other responses (Fig. 1). For the object-cued task, we defined goal-directed imitation as location matches in the Object conditions and motion matches in the No-Object condition. Other responses were motion matches in the Object conditions and location matches in the No-Object condition. For the vocal-cued task, we defined goal-directed imitation as copying intentional actions. Copying accidental actions were considered as other responses. For each task, a proportion was calculated by dividing the number of goal-directed imitations by the number of total responses (goal-directed imitation and other responses). For both tasks, a number above 0.5 indicated a greater number of goal imitation, compared with other imitation, was performed. A number of 0.5 indicated that an equal number of goal imitation and other imitation was performed. A number below 0.5 indicated that a greater number of other imitation, compared with goal imitation, was performed (see Fig. 1). The mean proportion in the object-cued task was .60 $(S D=.47)$ and in the vocal-cued task was $.52(S D=.18)$. A Pearson correlation between the proportion of goal-directed imitation in the object-cued task and the proportion of goal-directed imitation in the vocal-cued task revealed a significant positive relation between performances on the two tasks, $r=.46, p<.01$.

The results of the correlational analysis suggest that goal-directed imitation is related across different tasks; infants who performed more goal-directed imitation on one task also performed more on the other task and vice versa. A follow-up analysis was conducted to further investigate the relationship between goal-directed imitation on the two tasks. For this analysis, infants were divided into two groups based on their goal-directed imitation scores for the object-cued task at 13 months. Infants with proportional scores above 0.5 were considered as early goal-directed $(n=21)$. Infants who scored 0.5 or below were considered as not goal-directed $(n=16)$. A one-way ANOVA was conducted with early goal-directed versus not goal-directed as the independent factor and with the proportional score for goal imitation on the vocal-cued task at 14 months as the dependent factor. The results revealed that early goal-directed infants $(M=.59, S D=.18)$ demonstrated significantly more goal-directed imitation than not goal-directed infants $(M=.43, S D=.14)$ on the vocally cued task at 14 months,

Table 1

Longitudinal depiction of proportions of goal-directed imitation performed by infants in the object-cued task and vocal-cued task.

\begin{tabular}{lllr}
\hline Vocal-cued task & Object-cued task & \\
\cline { 2 - 4 } & Below 0.5 & At 0.5 & Above 0.5 \\
\hline Below 0.5 & 9 & 1 & 5 \\
At 0.5 & 2 & 1 & 3 \\
Above 0.5 & 2 & 1 & 13 \\
\hline
\end{tabular}

Note. The table displays the numbers of infants who exhibited these proportion patterns. A proportion above 0.5 indicates a greater number of goal imitation, a proportion below 0.5 indicates a greater number of other imitation, and a proportion at 0.5 indicates an equal number of goal imitation and other imitation. 
$F(1,35)=8.90, p<.01$. The results of this second analysis indicate that infants who demonstrated goaldirected imitation at 13 months were more likely to demonstrate it again at 14 months despite the differences in physical objects, goals, and communicative cues across the tasks (see Table 1).

\section{Additional analyses}

To address whether the observed stability in goal-directed imitation was due to a third factor, such as infants' attention to or engagement with the tasks, we coded infant looking throughout demonstrations in both tasks. We coded infant looking as looking at the demonstration or looking elsewhere and then calculated the percentage of time spent looking to the demonstration for each infant on each task. Pearson correlations examined relations between the continuous variable looking to demonstration and the continuous variable proportion of goal-directed imitation in each equivalent trial (three trials for object-cued task and eight trials for vocal-cued task). The results revealed no significant relations ( $p$ values $>.14)$.

\section{Discussion}

Numerous studies have reported that infants view others as intentional agents and that this understanding guides their imitative behavior, leading infants to selectively imitate the goals and intentions of others. We compared this account of imitation against alternative accounts proposing that imitation does not involve inferences about goals and intentions but instead results from learning about specific object affordances and outcomes or attention to specific communicative cues. To do so, we identified two distinct imitation tasks that involved different objects, goals, and cues, and we administered them at a transitional age when, according to previous studies, infants should be expected to exhibit individual differences (Carpenter et al., 1998, 2005; Sakkalou \& Gattis, 2012). This allowed us to first examine performance on each task and then examine the relation between performances across tasks. The proposal that imitation relies on a general ability to make inferences about intentions predicts that performance should be related across the two tasks despite the dissimilarities in task features and structure. The proposal that imitation instead reflects infant responses to object affordances or communicative cues does not predict stability across the two tasks because performance across tasks should be more independent.

The results of the object-cued task were consistent with those of Carpenter and colleagues (2005); at the group level, 13-month-olds imitated selectively, matching the location of the experimenter's actions when objects such as houses or towers were present and matching the experimenter's specific motion when no objects were present. This was not due to learning across trials or to a preference for a specific response. In all three conditions, infants observed identical actions but copied different aspects of the demonstration depending on the presence of target objects, thereby demonstrating selective imitation of action goals. Importantly, in our study, all infants were included in the analyses, allowing stability of infant behavior to be examined.

The results of the vocal-cued task were consistent with those of previous studies using a wider age range; at the group level, 14-month-olds were not more likely to copy intentional actions rather than accidental actions. Previous studies (Olineck \& Poulin-Dubois, 2005; Sakkalou \& Gattis, 2012) reported that at the group level 14- to 18-month-olds copy intentional actions more than accidental actions but that infants were less likely to do so before 16 months, indicating that the ability is still in a transitional period at 14 months and may continue to develop during subsequent months or even years (Gardiner, Greif, \& Bjorklund, 2011). As with the object-cued task, performance was not due to learning across trials or to a preference for a specific response.

Performance across the two tasks was related, supporting the claim that imitation reflects a general ability to copy intentions and goals. Two analyses demonstrated that goal-directed imitation on the object-cued task at 13 months was positively related to goal-directed imitation on the vocal-cued task at 14 months. Much like Sommerville and Woodward's (2005) study, in which infants who identified goal-directed actions in others were also better able to perform goal-directed actions themselves, the stability in individual infants' performances in our study suggests that despite the dissimilarities 
across the tasks, infants were relying on a general ability to interpret others' behavior in terms of intentions and not simply learning about specific outcomes or attention to specific cues.

These results provide the first empirical evidence of stability in goal-directed imitation. Few studies have examined individual differences in imitation, and even fewer have done so longitudinally (but see Fenstermacher \& Saudino, 2006, 2007). Olineck and Poulin-Dubois (2005) compared 14-month-olds' imitation across Meltzoff's (1995) failed attempts task and a variation on the vocalcued task described above, but they found no relation between the two tasks. They suggested that the two tasks might draw on different skills, pointing to the presence versus absence of communicative cues in the vocal-cued task and the failed attempts task. The significant positive relation we observed between imitation on an object-cued task that did not manipulate communicative cues and a vocal-cued task that did indicates, however, that this is not the case. It may be that completing an incomplete action relies on learning about outcomes rather than intentions, as argued by Huang and colleagues (2002), and for this reason performance is not related to goal-directed imitation.

Our longitudinal design examined performance across two age points just 1 month apart, but future studies might examine stability across longer periods. Most studies of imitation have tested just one age group or used a cross-sectional design in which each infant is tested at just one age. Further studies using longitudinal designs are needed to investigate explanatory hypotheses about imitation, including the relations between imitation and other aspects of social cognition. Several studies using longitudinal methods to investigate social cognition have demonstrated continuity in children's sensitivity to and inferences about mental states (e.g., Aschersleben, Hofer, \& Jovanovic, 2008; Wellman, Phillips, Dunphy-Lelii, \& LaLonde, 2004). The results of these studies suggest that mental state understanding is a general process that is used across a wide range of tasks. More research is needed to examine the developmental progression from social understanding during early infancy to more mature forms of mental state reasoning such as false belief. Future research might also investigate how this progression is influenced by the broader social environment.

\section{Acknowledgments}

This research was supported by the Leverhulme Trust and the Economic and Social Sciences Research Council. We thank Barbara Carotti for her help with coding of the data and Sylwia Matuszewska for her help during the monthly participant meetings. We thank Malinda Carpenter and Ulrike Hahn for useful discussions during this project.

\section{References}

Aschersleben, G., Hofer, T., \& Jovanovic, B. (2008). The link between infant attention to goal-directed action and later theory of mind abilities. Developmental Science, 11, 862-868.

Baldwin, D. A., Baird, J. A., Saylor, M. M., \& Clark, M. A. (2001). Infants parse dynamic action. Child Development, 72, $708-717$.

Bekkering, H., Wohlschläger, A., \& Gattis, M. (2000). Imitation of gestures in children is goal-directed. Quarterly Journal of Experimental Psychology A, 53, 153-164.

Brugger, A., Lariviere, L. A., Mumme, D. L., \& Bushnell, E. W. (2007). Doing the right thing: Infants' selection of actions to imitate from observed event sequences. Child Development, 78, 806-824.

Carpenter, M., Akhtar, N., \& Tomasello, M. (1998). Fourteen- to 18-month-old infants differentially imitate intentional and accidental actions. Infant Behavior and Development, 21, 315-330.

Carpenter, M., Call, J., \& Tomasello, M. (2005). Twelve- and 18-month-olds copy actions in terms of goals. Developmental Science, 8, F13-F20.

Ellis-Davies, K., Sakkalou, E., Fowler, N. C., Hilbrink, E. E., \& Gattis, M. (in press). CUE: The continuous unified electronic diary method. Behavior Research Methods. http://dx.doi.org/10.3758/s13428-012-0205-1.

Fenstermacher, S. K., \& Saudino, K. J. (2006). Understanding individual differences in young children's imitative behavior. Developmental Review, 26, 346-364.

Fenstermacher, S. K., \& Saudino, K. J. (2007). Toddler see, toddler do? Genetic and environmental influences on laboratoryassessed elicited imitation. Behavior Genetics, 37, 639-647.

Gardiner, A. K., Greif, M. L., \& Bjorklund, D. F. (2011). Guided by intention: Preschoolers' imitation reflects inferences of causation. Journal of Cognition and Development, 12, 355-373.

Gattis, M., Bekkering, H., \& Wohlschläger, A. (2002). Goal-directed imitation. In A. Meltzoff \& W. Prinz (Eds.), The imitative mind (pp. 183-205). Cambridge, UK: Cambridge University Press.

Gergely, G., \& Csibra, G. (2005). The social construction of the cultural mind: Imitative learning as a mechanism of human pedagogy. Interaction Studies, 6, 463-481. 
Huang, C., Heyes, C., \& Charman, T. (2002). Infants' behavioral re-enactment of "failed attempts": Exploring the role of emulation learning, stimulus enhancement, and understanding of intentions. Developmental Psychology, 38, 840-855.

Kiraly, I. (2009). The effect of the model's presence and of negative evidence on infants' selective imitation. Journal of Experimental Child Psychology, 102, 14-25.

Meltzoff, A. N. (1995). Understanding the intentions of others: Re-enactment of intended acts by 18-month-old children. Developmental Psychology, 31, 838-850.

Nielsen, M. (2009). 12-month-olds produce others' intended but unfulfilled acts. Infancy, 14, 377-389.

Olineck, K., \& Poulin-Dubois, D. (2005). Infants' ability to distinguish between intentional and accidental actions and its relation to internal state language. Infancy, 8, 91-100.

Phillips, A., Wellman, H., \& Spelke, E. (2002). Infants' ability to connect gaze and emotional expression as cues to intentional action. Cognition, 85, 53-78.

Sakkalou, E., \& Gattis, M. (2012). Infants infer intentions from prosody. Cognitive Development, 27, 1-16.

Sommerville, J. A., \& Woodward, A. L. (2005). Pulling out the intentional structure of human action: The relation between action production and processing in infancy. Cognition, 95, 1-30.

Wellman, H. M., Phillips, A. T., Dunphy-Lelii, S., \& LaLonde, N. (2004). Infant social attention predicts preschool social cognition. Developmental Science, 7, 283-288.

Woodward, A. L. (1998). Infants selectively encode the goal object of an actor's reach. Cognition, 69, 1-34. 